\author{
Marquette University \\ e-Publications@Marquette
}

$1-2020$

\title{
Enhanced Light Harvesting Ability in Zeolitic Imidazolate Frameworks Through Energy Transfer from CdS Nanowires
}

Yixuan Zhou

Marquette University

Wenhui Hu

Marquette University

Sizhuo Yang

Marquette University

Jier Huang

Marquette University, jier.huang@marquette.edu

Follow this and additional works at: https://epublications.marquette.edu/chem_fac

Part of the Chemistry Commons

\section{Recommended Citation}

Zhou, Yixuan; Hu, Wenhui; Yang, Sizhuo; and Huang, Jier, "Enhanced Light Harvesting Ability in Zeolitic Imidazolate Frameworks Through Energy Transfer from CdS Nanowires" (2020). Chemistry Faculty Research and Publications. 1024.

https://epublications.marquette.edu/chem_fac/1024 
Marquette University

e-Publications@Marquette

\section{Chemistry Faculty Research and Publications/College of Arts and Sciences}

This paper is NOT THE PUBLISHED VERSION.

Access the published version via the link in the citation below.

Physical Chemistry Chemical Physics, Vol. 22, No. 7 (January 2020): 3849-3854. DOI. This article is (C) Royal Society of Chemistry and permission has been granted for this version to appear in $\underline{\text { e- }}$ Publications@Marquette. Royal Society of Chemistry does not grant permission for this article to be further copied/distributed or hosted elsewhere without the express permission from Royal Society of Chemistry.

\section{Enhanced Light Harvesting Ability in Zeolitic Imidazolate Frameworks Through Energy Transfer from CdS Nanowires}

Yixuan Zhou

School of Applied Physics and Materials, Wuyi University, Jiangmen, China and Department of Chemistry, Marquette University, Milwaukee, Wisconsin

Wenhui Hu

Department of Chemistry, Marquette University, Milwaukee, Wisconsin

Sizhuo Yang

Department of Chemistry, Marquette University, Milwaukee, Wisconsin

Jier Huang

Department of Chemistry, Marquette University, Milwaukee, Wisconsin

\section{Abstract}

Zeolitic imidazolate frameworks (ZIFs) represent a novel class of porous crystalline materials that have demonstrated potential as light harvesting materials for solar energy conversion. In order to facilitate their application in solar energy conversion, it is necessary to expand their absorption further into the realm of the 
solar spectrum. In this work, we report the incorporation of semiconductor cadmium sulfide nanowires (CdS NWs) into ZIF-67 (CdS@ZIF-67), where a broader region of the solar spectrum can be absorbed by CdS NWs and relayed to ZIF-67 through an energy transfer (EnT) process. Using steady-state emission and time resolved emission and absorption spectroscopy, we show that efficient EnT can occur from CdS NWs to ZIF-67 by selective excitation of CdS NWs. The EnT time is $~ 729.9$ ps, which corresponds to $71.2 \%$ EnT efficiency.

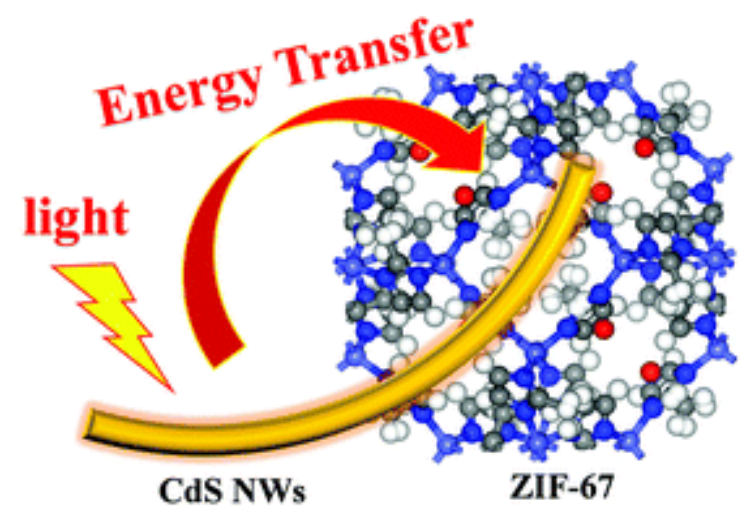

\section{Introduction}

Zeolitic imidazolate frameworks (ZIFs) represent a subclass of metal organic frameworks (MOFs) comprising metal ions tetrahedrally coordinated by imidazolate linkers. ${ }^{1-3}$ The inherent porous nature, high surface area, and tunable cavities of these materials have led to their various applications in gas separation and storage, ${ }^{1,4-}$ ${ }^{7}$ chemical sensors, ${ }^{8,9}$ and catalysis. ${ }^{10-13}$ In addition to these applications, recent work on ZIFs has extended their potential applications into the realm of solar energy conversion. For example, ZIFs have been used as photocatalysts for visible light-driven water splitting and $\mathrm{CO}_{2}$ reduction. ${ }^{14-20}$ Recently, our studies on ZIF-67 frameworks, synthesized from $\mathrm{Co}\left(\mathrm{NO}_{3}\right)_{2}$ and 2-methyl imidazolate, showed that these materials not only possess broad absorption in the UV-visible-near IR region but also exhibit a long-lived excited state (ES) (2.9 $\mu$ s lifetime) with charge separation (CS) character. ${ }^{16}$ Recent experiments on the effect of ZIF structure on ES dynamics uncovered that the porous framework of ZIFs plays a central role in the formation of the long-lived CS state and that metal-to-metal charge transfer is a contributing mechanism to $\mathrm{CS}^{21} \mathrm{~A}$ further study showed that the electron in this CS state can be extracted through interfacial electron transfer (ET) from excited ZIF to an organic species, ${ }^{22}$ providing crucial insights into the ability of ZIFs as intrinsic light harvesting and CS materials for solar energy conversion.

Despite these studies, which demonstrate the potential applications of ZIFs in solar energy conversion, the efficiency of harvesting solar energy based on ZIFs alone is anticipated to be low due to their limited absorption window in the solar spectrum and small extinction coefficient of metal $d-d$ transitions in the visible and near IR region. ${ }^{23}$ In order to achieve their application in solar energy conversion, it is necessary to further enhance the light harvesting ability of photoactive ZIFs. One desirable approach to enhance their light harvesting capability is to encapsulate/incorporate another light absorbing component (guest unit) with a large extinction coefficient and broad absorption that compensates the absorption spectrum of ZIFs. In such a way, additional solar energy can be harvested by the guest unit and relayed to ZIFs through an energy transfer (EnT) process. Following this strategy, we have recently achieved encapsulation of a molecular chromophore (RuN3) into the ZIF-67 framework, ${ }^{20}$ which shows efficient EnT from excited RuN3 to ZIF-67 with $86.9 \%$ efficiency, strongly supporting the promise of this strategy to enhance the light harvesting ability of ZIFs.

In addition to molecular chromophores, semiconductor nanocrystals are another important family of chromophores in solar energy conversion. Semiconductor nanocrystals typically have a large absorption extinction coefficient and strong emission in the broad region of the solar spectrum and can thus facilitate 
absorption of sunlight. ${ }^{24-29}$ In addition, their surfaces can be readily modified by altering the polarity of the ligands to control their solubility in preferred solvents, and by varying the length of ligands to control the distance between nanocrystals and ZIFs. ${ }^{30-36}$ In this work, we report the incorporation of semiconductor cadmium sulfide (CdS) nanowires (NWs) as the guest unit chromophore into the ZIF-67 framework, which forms a hybrid structure (CdS@ZIF-67). CdS NWs were chosen because the absorption of CdS NWs compensates the absorption spectrum of ZIF-67 and their emission has sufficient spectral overlap with the absorption of ZIF-67 to allow EnT to occur. Using the combination of steady state and time-resolved emission and absorption spectroscopy, we show that efficient EnT can occur from CdS NWs to ZIF-67 by selectively exciting CdS NWs.

\section{Materials and methods}

\section{Materials}

Cobalt(ii) nitrate hexahydrate $\left(\mathrm{Co}\left(\mathrm{NO}_{3}\right)_{2} \cdot 6 \mathrm{H}_{2} \mathrm{O}, 98-100 \%\right.$, Alfa Aesar), zinc nitrate hexahydrate $\left(\mathrm{Zn}\left(\mathrm{NO}_{3}\right)_{2} \cdot 6 \mathrm{H}_{2} \mathrm{O}\right.$, $99 \%$, Alfa Aesar), cadmium acetate $\left(\mathrm{Cd}\left(\mathrm{CH}_{3} \mathrm{COO}\right)_{2} \cdot 2 \mathrm{H}_{2} \mathrm{O}, 98 \%\right.$, Sigma-Aldrich), sodium diethyldithiocarbamate trihydrate $\left(\mathrm{NaS}_{2} \mathrm{CNEt}_{2} \cdot 3 \mathrm{H}_{2} \mathrm{O}, 98 \%\right.$, Alfa Aesar), 2-methylimidazole (99\%, Alfa Aesar), ethylenediamine $\left(\mathrm{C}_{2} \mathrm{H}_{8} \mathrm{~N}_{2}\right.$, 99\%, Acros Organics), 1-dodecanethiol ( $\mathrm{C}_{12} \mathrm{H}_{26} \mathrm{~S}, 98 \%$, Acros Organics), ethanol (anhydrous, Decon), and methanol (99.8\%, Alfa Aesar) were obtained as indicated.

\section{The synthesis of CdS NWs and CdS@ZIF-67 hybrid}

The synthesis of CdS NWs CdS NWs were synthesized according to a previously published procedure with minor modification. ${ }^{37}$ Briefly, $1 \mathrm{mmol}$ of $\mathrm{Cd}\left(\mathrm{CH}_{3} \mathrm{COO}\right)_{2} \cdot 2 \mathrm{H}_{2} \mathrm{O}$ and $2 \mathrm{mmol}$ of $\mathrm{NaS}_{2} \mathrm{CNEt}_{2} \cdot 3 \mathrm{H}_{2} \mathrm{O}$ were added into a $50 \mathrm{ml}$ Teflon-lined autoclave that had been filled with a mixed solvent of ethylenediamine $(17 \mathrm{ml})$ and dodecanethiol $(3 \mathrm{ml})$. The autoclave was kept in an oven at $180^{\circ} \mathrm{C}$ for 24 hours, and then allowed to cool down to room temperature naturally. A yellow precipitate was then collected by centrifugation and washed with ethanol and deionized water several times.

To prepare polyvinylpyrrolidone (PVP)-stabilized CdS NWs, the above-obtained CdS NWs were dispersed in 10 $\mathrm{ml}$ of an methanol solution with $250 \mathrm{mg}$ of PVP, followed by stirring for 24 hours. The sample was collected by centrifugation and washed with methanol several times to remove excess PVP. Finally, the PVP-stabilized CdS NWs were redispersed in methanol. The thin films of CdS NWs were prepared by spin coating CdS NWs onto glass substrates for optical measurements.

The synthesis of ZIF-67 and ZIF-8 thin films ZIF-67 and ZIF-8 were synthesized at room temperature according to previously published literature. ${ }^{38,39}$ Briefly, $5 \mathrm{ml}$ of 2-methylimidazole $(80 \mathrm{mM})$ and $3 \mathrm{ml}$ of $\mathrm{Co}\left(\mathrm{NO}_{3}\right)_{2} \cdot 6 \mathrm{H}_{2} \mathrm{O}(20$ $\mathrm{mM}$ ) or $\mathrm{Zn}\left(\mathrm{NO}_{3}\right)_{2} \cdot 6 \mathrm{H}_{2} \mathrm{O}(20 \mathrm{mM})$ were mixed in a glass vial. The mixture was kept at room temperature for 24 hours without stirring. ZIF-67 or ZIF-8 crystals were collected by centrifugation and washed with methanol several times. The thin films of ZIF-67 or ZIF-8 were prepared by immersing a piranha-etched glass substrate into the above mixed solution for $1 \mathrm{~h}$. The as-prepared film was rinsed with methanol to remove excess raw materials and dried in air for further use.

The synthesis of CdS@ZIF-67 and CdS@ZIF-8 thin films CdS@ZIF-67 hybrids were synthesized according to the published literature procedure with minor modification. ${ }^{40} \mathrm{In}$ a typical procedure, $1 \mathrm{ml}$ of PVP-stabilized CdS NW solution, $5 \mathrm{ml}$ of 2-methylimidazole $(80 \mathrm{mM})$ and $3 \mathrm{ml}$ of $\mathrm{Co}\left(\mathrm{NO}_{3}\right)_{2} \cdot 6 \mathrm{H}_{2} \mathrm{O}(20 \mathrm{mM})$ were mixed in a glass vial and then kept at room temperature for 24 hours without stirring. The formed product was collected by centrifugation and washed with methanol several times. The final product was re-suspended in methanol to protect it from air. CdS@ZIF-8 composites were synthesized with a similar procedure where $\mathrm{Co}\left(\mathrm{NO}_{3}\right)_{2} \cdot 6 \mathrm{H}_{2} \mathrm{O}$ was replaced by $\mathrm{Zn}\left(\mathrm{NO}_{3}\right)_{2} \cdot 6 \mathrm{H}_{2} \mathrm{O}$. The films of CdS@ZIF-67 or CdS@ZIF-8 were prepared by immersing a piranhaetched substrate into the above mixed solution for $1 \mathrm{~h}$. The as-prepared film was rinsed by methanol to remove 
excess raw materials and dried in air before characterization. The procedure of the synthesis can be seen in Scheme 1.

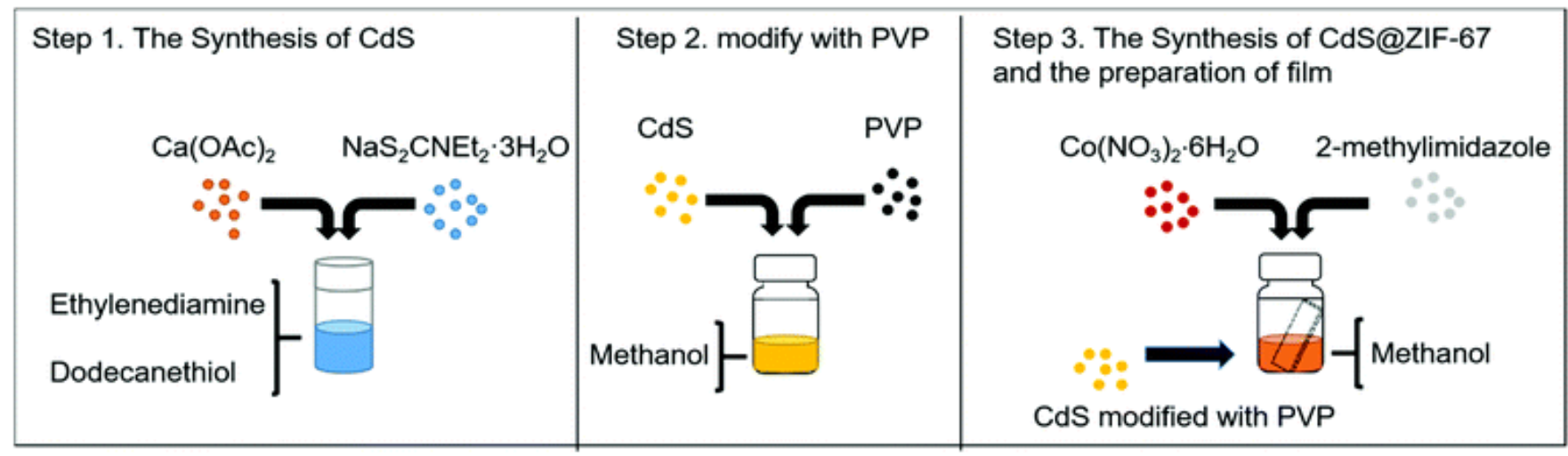

Scheme 1 The synthetic scheme of CdS NWs capped with PVP and CdS@ZIF-67 thin film.

\section{Characterization methods}

Standard characterization UV-Visible diffuse reflectance spectra were obtained using a Cary 5000 UV-VIS-NIR spectrophotometer with an internal diffuse reflectance accessory. The XRD patterns were obtained using a Rigaku Miniflex II XRD diffractometer with $\mathrm{Cu}$ K $\alpha$ radiation. All samples were dried in an oven at $70{ }^{\circ} \mathrm{C}$ for 5 hours before XRD measurement to remove methanol. The SEM images were collected by a JEOL JSM 6510-LV SEM system. The emission spectra and time-resolved fluorescence spectra were measured using a Photon Technology International (PTI) QM40 Quanta Master system equipped with a PicoMaster time correlated singlephoton counting (TCSPC) spectrofluorometer. The emission spectra of thin film samples were measured following $400 \mathrm{~nm}$ excitation.

Femtosecond absorption spectroscopy The femtosecond transient absorption (fs-TA) spectra were collected through a regenerative amplified Ti-sapphire laser system (Solstice; $800 \mathrm{~nm},<100 \mathrm{fs}$ FWHM, $3.5 \mathrm{Mj}$ per pulse, and $1 \mathrm{kHz}$ repetition rate). Seventy-five percent of the $800 \mathrm{~nm}$ fundamental pulse was introduced into TOPAS to generate a tunable pump wavelength. Twenty-five percent of the fundamental pulse was used to generate the visible probe $(450-750 \mathrm{~nm})$ using a sapphire window. A helios ultrafast spectrometer (Ultrafast Systems, LLC) was employed to record the TA spectra. All measurements were performed at $0.1 \mathrm{~mW}$ of a $400 \mathrm{~nm}$ pump.

\section{Results and discussion}

$X$-ray diffraction (XRD) and scanning electron microscopy (SEM) were used to confirm the formation and morphology of the as-synthesized CdS@ZIF-67 hybrid. As shown in Fig. 1a, the XRD patterns of the CdS@ZIF-67 hybrid show diffraction peaks at $24.6^{\circ}(100), 26.4^{\circ}(002), 28.1^{\circ}(101), 36.6^{\circ}(102), 43.6^{\circ}(110), 47.9^{\circ}(103), 51.8^{\circ}$ (112), and $53^{\circ}(201)$, which match well with the characteristic peaks of CdS NWs (black plot in Fig.

1a), ${ }^{37}$ suggesting the presence of CdS NWs in the CdS@ZIF-67 hybrid. In addition, diffraction peaks were observed at $7.2^{\circ}(011), 10.2(002)^{\circ}, 12.5^{\circ}(112), 14.5(022)^{\circ}, 16.3(013)^{\circ}$ and $17.9(222)^{\circ}$, which are consistent with the XRD patterns of ZIF-67 (green plot in Fig. 1a), ${ }^{38,39}$ suggesting the successful formation of ZIF-67 in the CdS@ZIF-67 hybrid. In addition to CdS@ZIF-67, the CdS@ZIF-8 hybrid was also prepared and used as a control sample in this work because ZIF-8 has negligible absorption in the visible region and EnT from CdS NWs to ZIF-8 is thus not expected. The formation of the CdS@ZIF-8 hybrid was confirmed by XRD (blue plot in Fig. 1a), where its XRD patterns consist of all the diffraction peaks of CdS NWs and ZIF-8, suggesting the formation of the CdS@ZIF-8hybrid. 

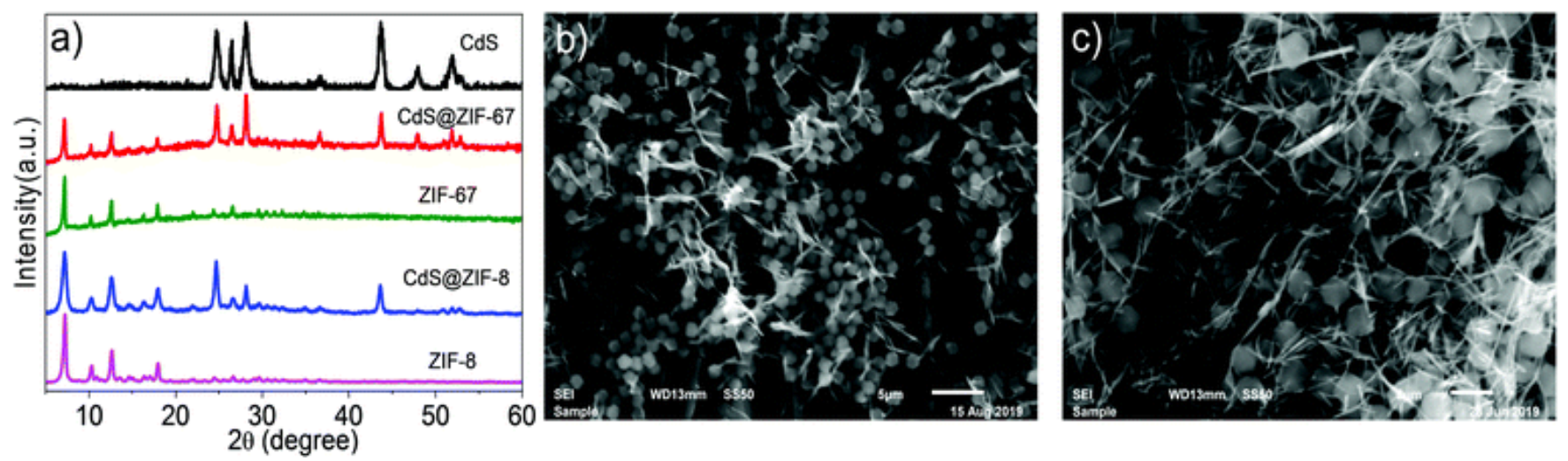

Fig. 1 (a) XRD patterns of the as-prepared CdS NWs, ZIF-67, ZIF-8, CdS@ZIF-67 and CdS@ZIF-8. SEM images of CdS@ZIF-8 (b) and CdS@ZIF-67 (c).

The morphology of CdS@ZIF-67 and CdS@ZIF-8 was examined by using SEM. As shown in Fig. 1b and c, the SEM images of both CdS@ZIF-67 and CdS@ZIF-8 display composites of wire-like CdS and rhombic dodecahedral shape crystals of ZIF-67 or ZIF-8 (Fig. S1, ESIT), further supporting the formation of CdS@ZIF-67 and CdS@ZIF-8 hybrids. The diameter of ZIF-8 and ZIF-67 is about 1-1.2 $\mu \mathrm{m}$ and the length of CdS NWs is about 5-6 $\mu \mathrm{m}$. While the majority of CdS NWs were grown on the surface of the ZIF crystals, some CdS NWs penetrated the ZIF crystals, which may facilitate interaction between CdS NWs and ZIF.

Fig. 2a shows the diffuse reflectance spectra of CdS@ZIF-67, CdS@ZIF-8, ZIF-8, ZIF-67, and CdS NWs. All optical measurements were performed on the as-prepared thin film samples. While ZIF- 8 has negligible absorption in the UV-visible region due to the $3 d^{10}$ electron configuration of $\mathrm{Zn}^{2+}, \mathrm{ZIF}-67$ displays a prominent transition at 540 $\mathrm{nm}, 564 \mathrm{~nm}$ and $588 \mathrm{~nm}$ corresponding to the ${ }^{4} \mathrm{~A}_{2}(\mathrm{~F}) \rightarrow{ }^{4} \mathrm{~T}_{1}(\mathrm{P})$ transition of tetrahedrally coordinated $\mathrm{Co}^{2+}$ and at $<350 \mathrm{~nm}$ corresponding to ligand to metal charge transfer. ${ }^{41,42}$ In the hybrid materials, an additional broad absorption band at 300-525 nm was observed in both CdS@ZIF-67 and CdS@ZIF-8. This band was observed in the CdS NWs, and it can be attributed to the bandgap transition of CdS NWs. ${ }^{43,44}$ These results further confirm the presence of both CdS NWs and ZIFs in the hybrid materials.
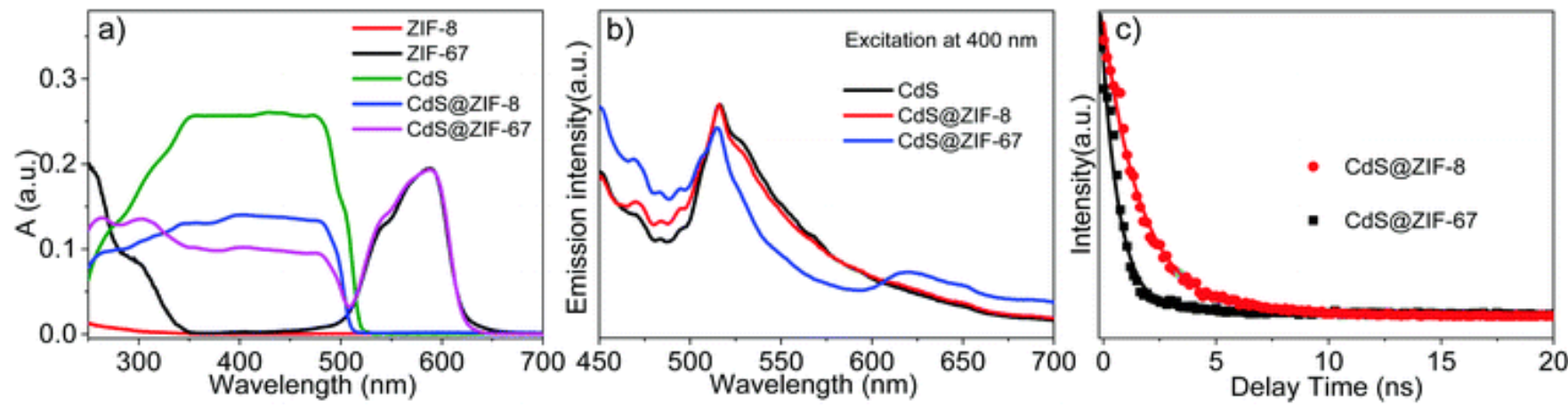

Fig. 2 (a) The diffuse reflectance spectra of ZIF-8, ZIF-67, CdS NWs, CdS@ZIF-67 and CdS@ZIF-8. (b) The emission spectra of CdS NWs, CdS@ZIF-8 and CdS@ZIF-67 following 400 nm excitation. (c) The comparison of emission lifetime decay kinetics of CdS@ZIF-8 and CdS@ZIF-67, which were collected at 515 nm after 400 nm excitation. Solid lines are the fits of the decays according to eqn (S1) (ESIt).

Fig. 2b compares the emission spectra of CdS@ZIF-8 and CdS@ZIF-67 following 400 nm excitation, which selectively excites CdS NWs. The emission spectrum of CdS@ZIF-8 (red plot) features a broad band centered at $525 \mathrm{~nm}$. This band resembles that of CdS NWs (black plot) and can thus be attributed to the emission from CdS NWs. While this emission band is also observed in the spectrum of CdS@ZIF-67, its intensity particularly on the red side shoulder is quenched, which is accompanied by the formation of a new emission band at $\sim 600-700 \mathrm{~nm}$. This new emission band was also observed upon direct excitation of ZIF-67 at $515 \mathrm{~nm}$ (Fig. S2, ESI ${ }^{\dagger}$ ) and can thus 
be attributed to the emission of ZIF-67. These results together suggest that EnT occurs from CdS NWs to ZIF-67, which results in the quenching of CdS emission and the observation of ZIF-67 emission. The emission quenching mainly occurs at the red side of the shoulder because this is the spectral region where CdS emission overlaps with the absorption of ZIF-67 (Fig. 2a).

The EnT process from CdS NWs to ZIF-67 was further confirmed by time resolved emission spectroscopy using the time correlated single photon counting (TCSPC) technique. As shown in Fig. 2c, the emission lifetime of CdS NWs at 515 nm is much shorter in CdS@ZIF-67 than in CdS@ZIF-8, suggesting the quenching of CdS emission by ZIF-67 due to the EnT process. To quantitatively evaluate the EnT process, the emission decay kinetics were fit with a biexponential decay function (eqn (S1), ESI†). The resulting fitting parameters are shown in Table S1

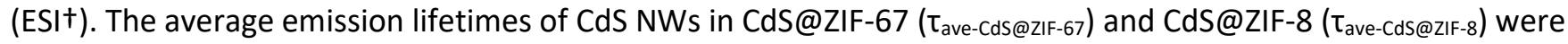
0.59 and 1.79 ns, respectively, resulting in an EnT time of 0.89 ns.

Due to the limited time resolution of our TCSPC ( 1 ns), we may have missed the early time kinetics of the EnT process. To gain insight into the early time kinetics of EnT from CdS NWs to ZIF-67, we examined the carrier dynamics of CdS NWs in CdS@ZIF-8 and CdS@ZIF-67 using femtosecond TA (fs-TA) spectroscopy ( 200 fs time resolution).Fig. 3a and b show the fs-TA spectra of CdS@ZIF-8 and CdS@ZIF-67 following 400 nm excitation, respectively. As shown in Fig. 3a, the fs-TA spectra of CdS@ZIF-8 consist of a negative feature centered around $490 \mathrm{~nm}$ and a positive absorption band $<470 \mathrm{~nm}$. The former can be attributed to the $1 \mathrm{~S}$ exciton bleach band in $\mathrm{CdS},{ }^{45}$ whereas the positive absorption band can be attributed to the Stark-effect-induced absorption resulting from the presence of $1 \mathrm{~S}$ exciton. ${ }^{46}$ Both features were also observed in the fs-TA spectra of CdS@ZIF-67 (Fig. 3b). However, compared to the spectra of CdS@ZIF-8, an additional feature was observed for CdS@ZIF-67 at 620 nm (inset of Fig. 3b), which can be attributed to the excited state of ZIF-67 resulting from EnT from CdS NWs. The EnT process is further confirmed from the comparison of the kinetic traces of $1 \mathrm{~S}$ exciton bleach recovery between CdS@ZIF-67 and CdS@ZIF-8. As shown in Fig.3c, the 1S exciton bleach recovery in CdS@ZIF-67 is significantly enhanced compared to that of CdS@ZIF-8, suggesting that the removal of the 1S electron from the conduction band of CdS NWs is faster in the former than in the latter. This further confirms the EnT process from CdS NWs to ZIF-67 as EnT results in faster recombination of electrons in the conduction band of CdS NWs with the holes in the valence band. These kinetic traces can be fit by a rise/decay function with one rising component and three decay components. The fitting parameters are listed in Table S2 (ESI + ) and the best fits to the kinetic traces are shown as solid lines in Fig. 3c. The rising component can be assigned to the relaxation of electrons from a higher energy level to the lowest energy level in the conduction band of CdS NWs. ${ }^{46}$ Among the three decay components, we were not able to accurately determine the long-time component $\left(\tau_{4}\right)$ because of the limited time window of our fs-TA spectrometer ( $5 \mathrm{~ns})$. Instead, we used the long-time component in the emission decay measurement ( $\tau_{2}$ in Table S1, ESI ${ }^{\dagger}$ ) as $\tau_{4}$. From these decay components, the average electronhole lifetimes for CdS@ZIF-67 and CdS@ZIF-8 were estimated to be 519.6 ps and 1803.2 ps, respectively, which led to an EnT time of $739.9 \mathrm{ps}$ and EnT efficiency of $71.1 \%$ calculated according to eqn (S2) and (S3) (ESI + ). ${ }^{47}$ The EnT time of $739.9 \mathrm{ps}$ is close to (slightly faster than) that calculated according to emission decay (0.89 ns), suggesting the validity of both spectral methods in determining the EnT process. While the EnT efficiency is slightly lower than that in the RuN3 encapsulated ZIF-67 system ( $86.9 \%$ efficiency), ${ }^{20}$ it suggests the promise of the strategy to enhance the light harvesting ability of ZIFs by incorporating semiconductor chromophores into ZIF structures. 

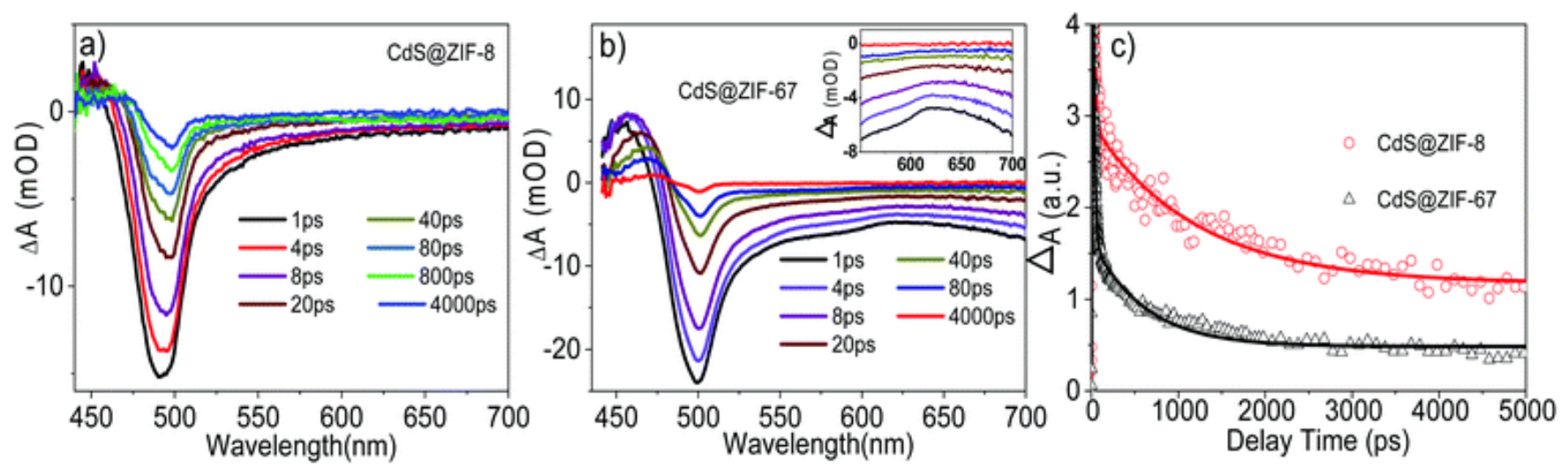

Fig. 3 Femtosecond transient absorption spectra of (a) ZIF-8@CdS and (b) ZIF-67@CdS. The inset shows the enlarged regions of (b) in the range of 550-700 nm. (c) Comparison of kinetic traces between ZIF-8@CdS and ZIF-67@CdS at 500 nm.

\section{Conclusions}

In this work, we have incorporated semiconductor CdS NWs into ZIF-67, which forms a hybrid, CdS@ZIF-67. Using steady state emission and time resolved emission and absorption spectroscopy, we show that efficient energy transfer can occur from CdS NWs to ZIF-67, suggesting the capability to enhance light harvesting ability of ZIF-67 through the energy transfer process from CdS NWs. This work further demonstrates the great potential of ZIFs as intrinsic light harvesting materials for solar energy conversion.

\section{Conflicts of interest}

There are no conflicts to declare.

\section{Acknowledgements}

This work was supported by the National Science Foundation (DMR-1654140) and ACS-PRF (57503-DNI6). Wenhui Hu acknowledges a John J. Eisch fellowship during the 2019-2020 academic year. Yixuan Zhou acknowledges a visiting scholar fellowship at Wuyi University for the year of 2018-2019.

\section{Notes and References}

A. Phan , C. J. Doonan , F. J. Uribe-Romo , C. B. Knobler, M. O’Keeffe and O. M. Yaghi , Acc. Chem. Res., 2010, $43,58-67$.

B. R. Pimentel , A. Parulkar , E. K. Zhou , N. A. Brunelli and R. P. Lively , ChemSusChem, 2014, 7 , 3202 -3240.

B. L. Chen , Z. X. Yang , Y. Q. Zhu and Y. D. Xia , J. Mater. Chem. A, 2014, 2, $16811-16831$.

K. G. Ray , D. L. Olmsted , J. M. R. Burton , Y. Houndonougbo , B. B. Laird and M. Asta , Chem. Mater., 2014, 26 , $3976-3985$.

H. Bux , A. Feldhoff , J. Cravillon, M. Wiebcke, Y.-S. Li and J. Caro , Chem. Mater., 2011, 23, 2262 -2269.

T. Wu , X. Feng , S. K. Elsaidi , P. K. Thallapally and M. A. Carreon , Ind. Eng. Chem. Res., 2017, 56 , 1682 -1686.

R. Banerjee , A. Phan , B. Wang , C. Knobler , H. Furukawa , M. Keeffe and O. M. Yaghi , Science, 2008, 319 , 939.

E.-X. Chen , H. Yang and J. Zhang , Inorg. Chem., 2014, 53, $5411-5413$.

W. Ma , Q. Jiang , P. Yu , L. Yang and L. Mao , Anal. Chem., 2013, 85, $7550-7557$.

J. Lee, O. K. Farha , J. Roberts, K. A. Scheidt , S. T. Nguyen and J. T. Hupp , Chem. Soc. Rev., 2009, 38 , $1450-$ 1459.

F. Zhang , Y. Wei , X. Wu , H. Jiang , W. Wang and H. Li , J. Am. Chem. Soc., 2014, 136, $13963-13966$.

U. P. N. Tran , K. K. A. Le and N. T. S. Phan , ACS Catal., 2011, 1, $120-127$.

T. T. Isimjan , H. Kazemian , S. Rohani and A. K. Ray , J. Mater. Chem., 2010, 20 , $10241-10245$. 
S. Wang and X. Wang , Appl. Catal., B, 2015, 162, $494-500$.

S. Wang , W. Yao , J. Lin , Z. Ding and X. Wang , Angew. Chem., 2014, 53 , $1034-1038$.

B. Pattengale , S. Yang , J. Ludwig , Z. Huang , X. Zhang and J. Huang , J. Am. Chem. Soc., 2016, 138, $8072-$ 8075 .

G. R. Jia , L. L. Liu , L. Zhang , D. T. Zhang , Y. Wang , X. Q. Cui and W. T. Zheng , Appl. Surf. Sci., 2018, 448 , 254 260.

Y. Liu , L. Deng , J. P. Sheng , F. Y. Tang, K. Zeng , L. Q. Wang , K. X. Liang , H. Hu and Y. N. Liu , Appl. Surf. Sci., $2019,498,143899$.

C. Y. Guo , J. Guo , Y. H. Zhang , D. Wang , L. Zhang , Y. Guo , W. L. Ma and J. D. Wang , CrystEngComm, 2018, 20 , $7659-7665$.

S. Yang , B. Pattengale, E. L. Kovrigin and J. Huang , ACS Energy Lett., 2017, 2 , $75-80$.

B. Pattengale , D. J. SantaLucia , S. Yang , W. Hu , C. Liu , X. Zhang , J. F. Berry and J. Huang , J. Am. Chem. Soc., 2018, $140,11573-11576$.

B. Pattengale and J. Huang, Phys. Chem. Chem. Phys., 2018, $20,14884-14888$.

C. Housecroft and A. Sharpe , Inorganic Chemistry, Pearson Education Ltd, Harlow UK, 2012,.

R. Peng , C.-M. Wu , J. Baltrusaitis , N. M. Dimitrijevic , T. Rajh and R. T. Koodali , Chem. Commun., 2013, 49, $3221-3223$.

J. Yu , Y. Yu , P. Zhou, W. Xiao and B. Cheng , Appl. Catal., B, 2014, 156-157, 184-191.

K. Wu , Z. Chen , H. Lv , H. Zhu , C. L. Hill and T. Lian , J. Am. Chem. Soc., 2014, 136, $7708-7716$.

L. J. Zhang , S. Li , B. K. Liu , D. J. Wang and T. F. Xie , ACS Catal., 2014, 4, $3724-3729$.

Z.-R. Tang , B. Han , C. Han and Y.-J. Xu , J. Mater. Chem. A, 2017, 5, $2387-2410$.

A. Kongkanand, K. Tvrdy, K. Takechi , M. Kuno and P. V. Kamat , J. Am. Chem. Soc., 2008, 130, 4007 -4015.

Z. B. Zhuang , X. T. Lu , Q. Peng and Y. D. Li , J. Am. Chem. Soc., 2010, 132 , $1819-1821$.

K. Murakoshi , H. Hosokawa, M. Saito , Y. Wada and S. Yanagida , J. Colloid Interface Sci., 1998, 203, 225 -228.

K. N. Lawrence, S. Dolai and R. Sardar , RSC Adv., 2014, 4 , $30742-30753$.

A. M. Coto-Garcia , M. T. Fernandez-Arguelles , J. M. Costa-Fernandez , A. Sanz-Medel, M. Valledor , J. C.

Campo and F. J. Ferrero, J. Nanopart. Res., 2013, 15 ,.

V. K. Gasparyan J. Fluoresc., 2014, $24,1433-1438$.

Z. A. Peng and X. G. Peng , J. Am. Chem. Soc., 2001, 123, $183-184$.

J. Lee and C. S. Han , Nanoscale Res. Lett., 2015, 10, 145 .

D. Xu , Z. Liu , J. Liang and Y. Qian , J. Phys. Chem. B, 2005, 109, 14344-14349.

M. Zhu , S. R. Venna , J. B. Jasinski and M. A. Carreon, Chem. Mater., 2011, 23, $3590-3592$.

Y. Guo , J. Tang , H. Qian , Z. Wang and Y. Yamauchi , Chem. Mater., 2017, 29 , 5566 -5573 .

G. Lu , S. Li , Z. Guo , O. K. Farha , B. G. Hauser , X. Qi , Y. Wang , X. Wang, S. Han , X. Liu , J. S. DuChene , H. Zhang , Q. Zhang , X. Chen , J. Ma , S. C. J. Loo , W. D. Wei , Y. Yang , J. T. Hupp and F. Huo , Nat. Chem., $2012,4,310-316$.

J. Qin , S. Wang and X. Wang , Appl. Catal., B, 2017, 209, 476-482

J. P. Lourenço , M. F. Ribeiro , C. Borges , J. Rocha , B. Onida , E. Garrone and Z. Gabelica , Microporous Mesoporous Mater., 2000, 38, 267 -278.

H. Choi and P. V. Kamat, J. Phys. Chem. Lett., 2013, 4, $3983-3991$.

H. Wang , S. B. Naghadeh , C. Li , V. L. Cherrette , P. Fang , K. Xu and J. Z. Zhang , J. Electrochem. Soc., 2019, 166 , H3146 - H3153

K. Wu , H. Zhu , Z. Liu , W. Rodríguez-Córdoba and T. Lian , J. Am. Chem. Soc., 2012, 134 , $10337-10340$

H. Zhu, N. Song and T. Lian , J. Am. Chem. Soc., 2010, 132, $15038-15045$.

S. Jin , H.-J. Son , O. K. Farha , G. P. Wiederrecht and J. T. Hupp , J. Am. Chem. Soc., 2013, 135 , $955-958$ 